7-15-2020

\title{
Lessons from a five-year research programme on FGM/C and their relevance for policy and programmes in Kenya
}

\section{Dennis Matanda}

Population Council

Agnes K. Meroka-Mutua

Samuel Kimani

Follow this and additional works at: https://knowledgecommons.popcouncil.org/departments_sbsr-rh

Part of the Demography, Population, and Ecology Commons, Family, Life Course, and Society Commons, Gender and Sexuality Commons, International Public Health Commons, and the Medicine and Health Commons How does access to this work benefit you? Let us know!

\section{Recommended Citation}

Matanda, Dennis, Agnes K. Meroka-Mutua, and Samuel Kimani. 2020. "Lessons from a five-year research programme on FGM/C and their relevance for policy and programmes in Kenya," Evidence to End FGM/C: Research to Help Girls and Women Thrive. New York: Population Council. 


\section{Lessons from a five-year research programme on FGM/C and their relevance for policy and programmes in Kenya}

The Evidence to End FGM/C programme consortium generates evidence to inform and influence investments, policies, and programmes for ending female genital mutilation/ cutting in different contexts.

Population Council

Lead Institution

Africa Coordinating Centre for the Abandonment of Female Genital Mutilation/Cutting, Kenya (ACCAF)

Global Research and Advocacy Group, Senegal (GRAG)

MannionDaniels Ltd. (MD)

Population Reference Bureau (PRB)

University of Washington (Prof. Bettina Shell-Duncan)

University of California, San Diego (Dr. Gerry Mackie)

Funded by

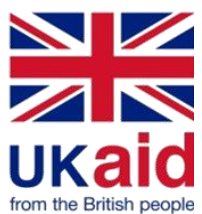

\section{Overview}

The Sustainable Development Goals (SDGs) target the elimination of all forms of harmful practises, including female genital mutilation/cutting $(\mathrm{FGM} / \mathrm{C})$ by 2030 . Kenya has made progress in addressing the elimination of $\mathrm{FGM} / \mathrm{C}$ through ratification of global instruments and enactment of a prohibition with the 2011 FGM Act and other supportive laws. The Act created the foundation for the establishment of the AntiFGM Board which provides policy leadership and coordination of antiFGM/C programmes in Kenya. More recently, the President of Kenya issued a decree for Figure 1. FGM/C prevalence by region accelerated elimination of (province) among women 15-49 yrs FGM/C by 2022. The Kenya Demographic Health Survey (KDHS) has been used to generate prevalence data on FGM/C since 1998. The 2014 $\mathrm{KDHS}$ estimates that nationally $21 \%$ of girls and women ages 15-49 years have undergone FGM/C - a decline from $38 \%$ in 1998. The overall prevalence masks both regional variations (see Figure 1) and ethnic differences. The prevalence of FGM/C remains high in some ethnic groups such as the Somali (94\%), Samburu (87\%), Kisii $(85 \%)$, and the Maasai (78\%).

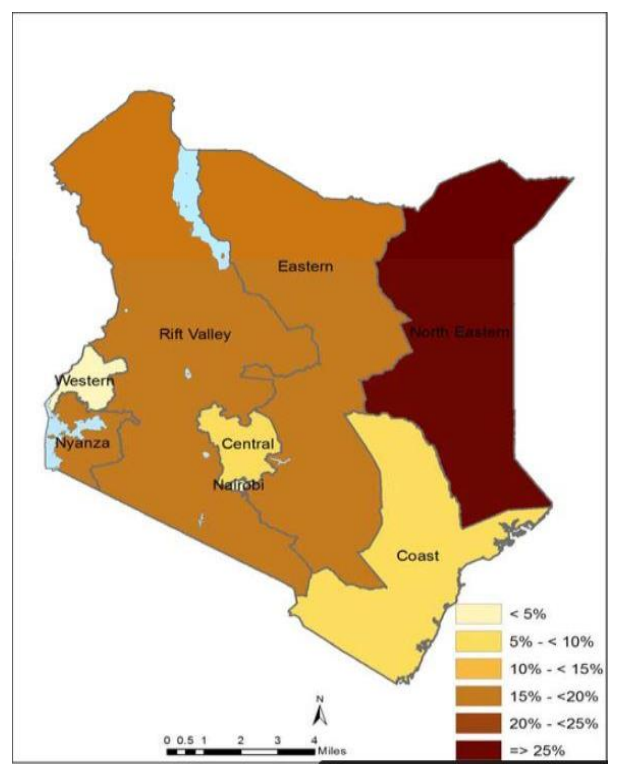

(Source: Shell-Duncan, Gathara, and Moore 2017)

As concerted efforts to end FGM/C gather speed, credible data is critical to effectively plan, develop policies, and implement programmes across sectors. This synthesis report highlights how evidence generated over the last five years by the Evidence to End FGM/C Research Programme can be used to inform policies and programmes to accelerate the total 
abandonment of FGM/C in Kenya. The report focuses on opportunities in governance, effective utilisation of the anti-FGM/C law, changes in practice and social norms associated with $\mathrm{FGM} / \mathrm{C}$, health system response to $\mathrm{FGM} / \mathrm{C}$, and the importance of data in accelerating $\mathrm{FGM} / \mathrm{C}$ abandonment.

\section{KEY LESSONS}

- There is a general decline in FGM/C prevalence, but the pattern of change is geographic- and ethnic-group-specific-some groups abandoning while others are not.

- The Kenyan governance structure at the national and county levels, as well as the existence of numerous nonstate actors, offer an opportunity to develop and adapt FGM/C programmes to fit local contexts.

- The existence of a legislative and policy framework that deals with FGM/C demonstrates a favourable political will and commitment to address FGM/C. There is a need to leverage on this framework through deliberate actions informed by evidence.

- Criminalisation of FGM/C has instilled fear but has not been effective in deterring people from practising FGM/C. Instead, criminalisation has driven the practice underground and to a younger age. Focus of the legal framework should be on regulation and crosssector interventions rather than over-relying on criminalisation.

- Research conducted in the last five years shows changes in FGM/C practise (e.g., cutting at younger ages, less severe forms of cutting, and cutting in secrecy), attitudes, behaviour, and the social norms associated with FGM/C (e.g., sexuality, marriageability, and culture). Therefore, programme implementers and policymakers need to pay attention to these changes when designing interventions in order to accelerate and support abandonment.

- The demand for medicalisation (FGM/C performed by health professionals) needs to be addressed with faith leaders, and the supply through measures to discourage health professionals from conducting FGM/C.

- The health system is inadequately prepared for FGM/C prevention and response. Health-system strengthening to address FGM/C should involve policy dissemination and development of protocol/tools; skill-building of health providers to better identify, manage, prevent and record FGM/C related complications; and integration of FGM/C into ongoing health programmes.

- WHO has published health costs to governments of treating health-related complications. These are only a part of the overall costs to society, which include psychological harm to girls and women, and loss of education and employment opportunities. The Kenyan government needs to allocate resources for both the prevention and the full consequences of FGM/C.

- The innovative use of national surveys can assist in pinpointing "FGM/C high spots." These data can be used to direct where resources should be targeted and effective local interventions implemented.

- Research is still needed into the potential for social media to play a role in ending FGM/C given its rapid expansion underway in Kenya. 


\section{Background}

Female genital mutilation/cutting (FGM/C) has continued to draw the attention of global, regional, and national policymakers because of its associated impacts on women's and girls' health and human rights. The global response to FGM/C is well articulated in the Sustainable Development Goals' key target for the elimination of all harmful practises by 2030, ICPD +25 commitments by governments, as well as various ratified international and regional legal/policy instruments (UN 2015). The instruments include General Recommendation 14 of the CEDAW Committee and Article 5 of the Additional Protocol to the African Charter on Human and Peoples' Rights on the Rights of Women in Africa call for states where FGM/C is practised to take measures towards the eradication of the practice (UN 1979).

Kenya has ratified the international and regional legal instruments, enacted the Prohibition of FGM Act of 2011 and other laws that contain provisions that address FGM/C including: the Children's Act, 2001 and Protection Against Domestic Violence Act, 2015 (ROK 2011). The Prohibition of FGM Act is a comprehensive piece of legislation that defines and criminalises FGM/C and other associated offences and prescribes the sanctions for those offences. The Act establishes the AntiFGM Board whose mandate is to offer policy leadership and programmatic coordination. The Board has developed policies, strategies, plans of actions, and tools that are critical in accelerating the abandonment of FGM/C. Among the documents developed by the Board is the National Policy for the Eradication of FGM (2019) which outlines focus areas for engagement in accelerating FGM/C abandonment (ROK 2019). These areas include: legal interventions, health, FGM/C data, changes in $\mathrm{FGM} / \mathrm{C}$ and emerging issues in $\mathrm{FGM} / \mathrm{C}$, evidence generation and utilisation, as well as continuous multi-stakeholder engagement. More recently, during the ICPD+25 meeting, the President of Kenya issued a decree for accelerated elimination of FGM/C by 2022. This policy leadership has given the much-needed impetus for multi-stakeholder strategies and roadmaps for addressing FGM/C, with the Anti-FGM Board leading the initiative.

Apart from the adoption of the law addressing FGM/C, Kenya is among the countries that included the FGM/C module in the Demographic and Health Survey (DHS), paving the way for the generation of credible data since 1998. These data have helped inform cross-sector strategic interventions, policies, programmes, and investments, as well as stimulating research. Data from the most recent (2014) Kenya Demographic and Health Survey (KDHS) estimates that $21 \%$ of girls and women ages 15-49 years have undergone FGM/C-a steady decline from 38\% in 1998 (KDHS 2014). Although the national prevalence has steadily declined over the years, the prevalence remains high in some ethnic groups such as the Somali (94\%), Samburu (87\%), Kisii (85\%), and the Maasai (78\%) (KDHS 2014). Figure 2 shows FGM/C prevalence across age cohorts among the six major ethnic groups that have traditionally conducted FGM/C. The graph shows FGM/C prevalence has substantially declined among the younger cohorts of Kalenjin, Kikuyu, and Meru ethnic groups. However, the prevalence remains very high among ethnic Somali women (across all age-cohorts), and older cohorts of Maasai and Kisii women, but appear to be declining in the youngest age cohorts (Shell-Duncan, Gathara, and Moore 2017).

Data and evidence on the magnitude and trends of FGM/C should also provide the economic impact of the practice for policymakers and programmers. Indeed, the economic dividends of saving girls from FGM/C as well as response to FGM/C-related complications can be calculated. Recently, the World Health Organisation (WHO) estimated that FGM/C could be costing world economies approximately USD 1.4 billion annually in health care for women living with conditions caused by FGM/C (WHO 2020). It is important to note that this figure includes only direct measurable health costs, but not the psychological, or the losses associated with girls leaving education or having reduced employment opportunities. 
Figure 2. FGM/C prevalence across age-cohorts by ethnicity

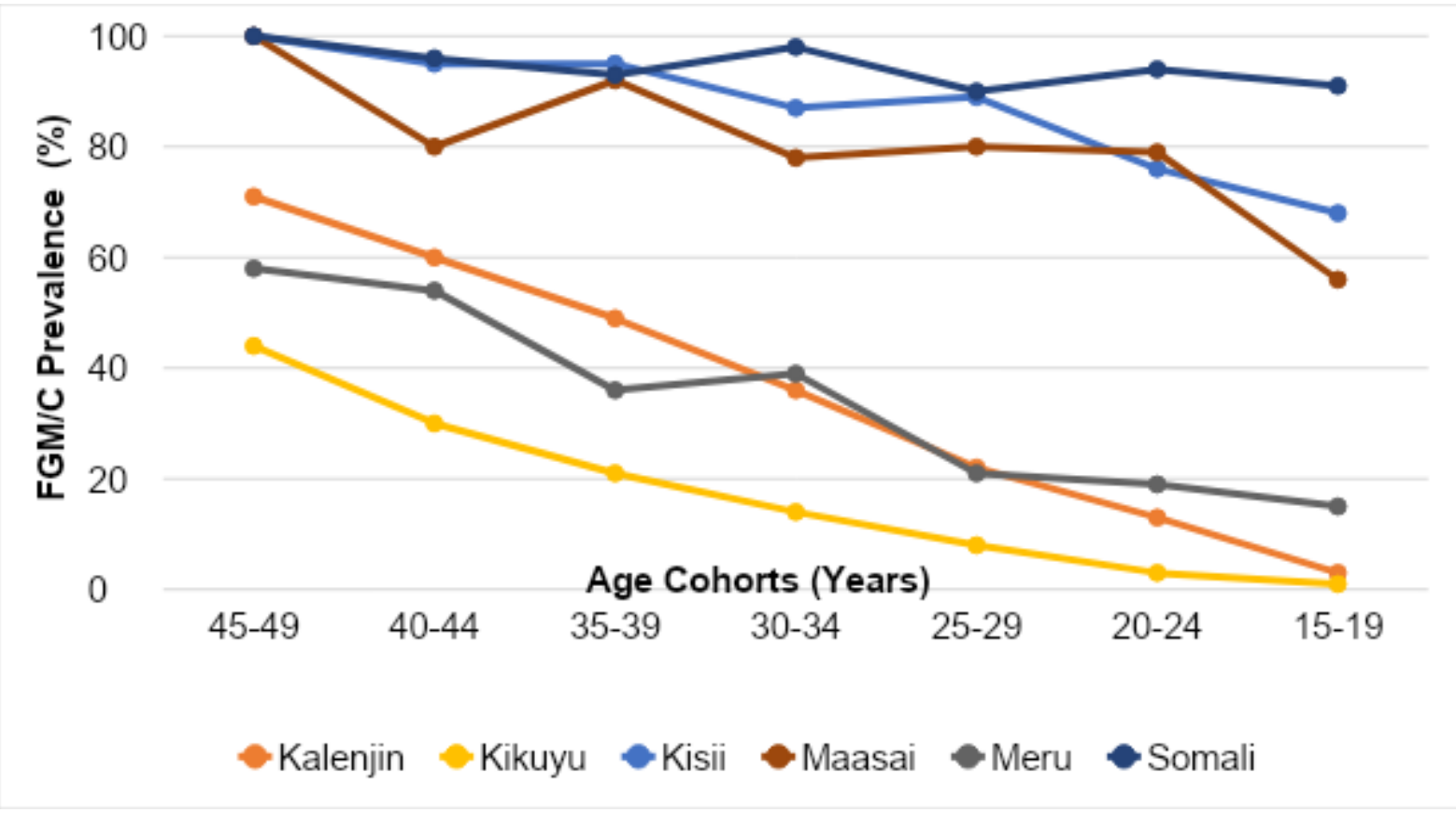

Source: Shell-Duncan, Gathara, and Moore 2017

As concerted efforts to end FGM/C gather speed, the key question is: How can we use evidence to inform policies and programmes across sectors to accelerate total abandonment of $F G M / C$ in Kenya? The Kenyan context presents opportunities for acceleration of abandonment but also challenges that need to be addressed. Specifically:

- The county government can play a pivotal role in spearheading change at the local level if its structures are optimally used;

- The passing of the anti-FGM/C law is a positive step in safeguarding the rights of women and girls but little is known about how the law should be effectively implemented to achieve the desired outcome;

- A myriad of interventions have been implemented; however more needs to be done to look at impact-if and how social norms and FGM/C practise are changing in response to these interventions;

- The health sector has a responsibility in promoting FGM/C abandonment and providing care to survivors, but we know little about existing gaps and opportunities; and

- Effective interventions to accelerate abandonment depend on availability of quality data and analysis, but more needs to be done to enable continuous generation and use of evidence.

This synthesis highlights the evidence generated from a five-year research programme on FGM/C in Kenya as part of the wider "Evidence to End FGM/C" Research Programme funded by DFID. The synthesis addresses: (i) emerging spaces and opportunities in relation to governance, (ii) utilisation of the anti-FGM law, (iii) changes in practise and social norms associated with $\mathrm{FGM} / \mathrm{C}$, (iv) health-system responses to $\mathrm{FGM} / \mathrm{C}$, (v) importance of collecting quality data in accelerating $\mathrm{FGM} / \mathrm{C}$ abandonment, (vi) structural barriers and interventions, and (vii) a reflection on emerging debates in Kenya around FGM/C. 


\section{Governance: Emerging Spaces and Opportunities}

During the period of the Evidence to End FGM/C programme, the role of governance structures in promoting $\mathrm{FGM} / \mathrm{C}$ interventions became increasingly apparent. While the programme did not set out to specifically examine the role of governance structures in ending FGM/C, the research projects interacted with the governance structures and drew important lessons outlined below:

\subsection{The role of devolved government in promoting the abandonment of FGM/C}

The constitution of Kenya provides a legal-governance structure comprising two distinct but interdependent government levels—national and 47 county governments (Kimani and Obianwu 2020). The Constitution places the responsibility of legal and policy formulation on the national government (ROK 2010, 28TooMany 2018). Therefore, nationally enacted laws and policies of nondevolved functions such as security and justice supersede any of those passed at the county level. Functions like health are devolved, but the mandate of policy formulation is placed at the national level. In the absence of sub-national legislation on a particular matter, the national law applies (Kimani and Obianwu).

This governance structure provides an opportunity for strengthening FGM/C interventions. For example, although the Prohibition of FGM Act of 2011 has vested the coordination of all antiFGM/C activities to the Anti-FGM Board, there is need for more coordinated functioning between the Board and county government structures. The 2019 National Policy on Eradication of FGM envisages a structure where the Anti-FGM Board works closely with county and sub-county AntiFGM Steering Committees (ROK 2019). The national government structures at the county level such as the county commissioner, the children's department, as well as their equivalent county government officials in different sectors should collaborate on matters related to FGM/C though working groups. This will ensure synergy, pooling of resources, accountability, and a united coherent approach to FGM/C abandonment. This calls for secondment of Anti-FGM Board officials to the counties.

\subsection{The role of stakeholder engagement and public participation}

The Constitution emphasises stakeholder engagement and public participation in developmental agenda-setting. Thus, devolved governance presents an opportunity through citizen/stakeholder engagement and participation for identification of priority programmes in the counties. Because of human rights violation and the adverse health outcomes of $\mathrm{FGM} / \mathrm{C}$ on the population, eradication of the practice should be identified as a priority area at the county level. For this to happen, citizens and community-based organisations (CBOs) that are well informed about FGM/C and its impact on health and human rights need to be involved. This involvement calls for training of CBOs on FGM/C as a health, social, and human rights problem that requires urgent attention by stakeholders, especially at the county-government level. Meaningful engagement will involve synthesis of the FGM/C-related evidence regarding a particular county which will enable counties where $\mathrm{FGM} / \mathrm{C}$ is prevalent to allocate funds from their annual budgets for anti-FGM/C activities.

\subsection{Implementation of community-based strategies}

The devolved governance structure presents an opportunity for implementation of communitybased approaches to address social norms underpinning FGM/C. Solutions to the gender inequalities in socioeconomic achievement should come from the communities themselves. This will encourage development of strategies based on better understanding of the cultural values and norms associated with the gender disparity, thus increasing their likelihood of implementation and acceptability. Additionally, this bottom-up approach with comprehensive community involvement 
has a greater likelihood of success compared with top-down approaches. Stakeholder engagement and understanding of community structures is needed.

\subsection{Existence of numerous non-state programmes}

At the national and county levels, there are a number of CBOs, nongovernmental organisations (NGOs), and civil societies that advance human rights and implement FGM/C interventions. Although the majority of these organisations may not have focused on FGM/C abandonment, they implement health, immunisation, HIV/AIDS, nutrition, and water sanitation, and hygiene (WASH) programmes that can be leveraged to implement FGM/C interventions, especially in the hard-toreach areas. This calls for concerted efforts to encourage collaboration and synergy across sectors and actors.

\subsection{Leveraging existing health programmes for integration of $\mathrm{FGM} / \mathrm{C}$}

Beyond NGOs, CBOs, and civil society, health systems have ongoing health workshops and sensitisation, maternal and child health programmes, nutrition, and school health programmes. Some are implemented through antenatal, postnatal, and family planning health service points. These service points can be entry points for responses to FGM/C through individualised message sharing and service provision such as sensitisation and counselling. Health providers involved in service provision in these delivery points may only require minimum orientation and training on $\mathrm{FGM} / \mathrm{C}$ interventions. For example, some FGM/C interventions such as health talks were reported in West Pokot-an indication that with resources they can be scaled up (Kimani and Okondo 2020). Additionally, programmes such as the Beyond Zero Campaign have infrastructure capacity such as ambulances and supplies for responding to maternal-child challenges that could also serve as a platform to roll out FGM/C interventions. The Beyond Zero programme is in the 47 counties and enjoys political good will.

\section{The Anti-FGM/C Law in Kenya: Lessons Learned}

Criminalisation of $\mathrm{FGM} / \mathrm{C}$ is intended to promote abandonment of the practice by acting as a deterrent. The 2011 Prohibition of FGM Act criminalises FGM/C and also creates other associated offences, which include aiding and abetting, being in possession of tools used for FGM/C, using one's premises for FGM/C, failing to report the commission of FGM/C, crossing the border to carryout $\mathrm{FGM} / \mathrm{C}$ in a different country, and using derogatory language to refer to an uncut woman or a man who marries an uncut woman. The Act provides for a mandatory minimum sentence of three years or a fine of 200,000 Kenya shillings for all offences under the Act, except where a victim of $\mathrm{FGM} / \mathrm{C}$ dies. In the case of the victim's death, the Act provides for a mandatory life sentence against the cutter. However, few cases have been prosecuted under the Prohibition of FGM Act since it was passed in 2011 (28TooMany 2018; UNFPA-UNICEF 2017), raising concerns about the enforcement and implementation of the law, as well as its effectiveness. In order to understand the motivations that people have for obeying the law, the Evidence to End FGM/C Research Programme conducted a study that looked at the moral, social, punitive, and religious areas of motivation for legal obedience (Meroka-Mutua, Mwanga, and Olungah 2020). This was a multi-country study, and in Kenya results showed that while the country has a robust legal framework for the prohibition of FGM/C through criminalisation, criminal sanctions have not worked as effectively as expected. In Kenya, the following findings were made concerning the anti-FGM law: 
3.1 Key findings on the implementation and enforcement of Kenya's anti-FGM/C law

The main motivating factor for people to obey the anti-FGM/C law in Kenya is the fear of penal sanctions. However, while criminalisation has instilled fear of the law, this has not had a strong deterrent effect. Figure 3 summarises the key barriers on implementation and enforcement of the anti-FGM/C law.

Figure 3. Key barriers limiting effective implementation and enforcement of the Anti-FGM/C law

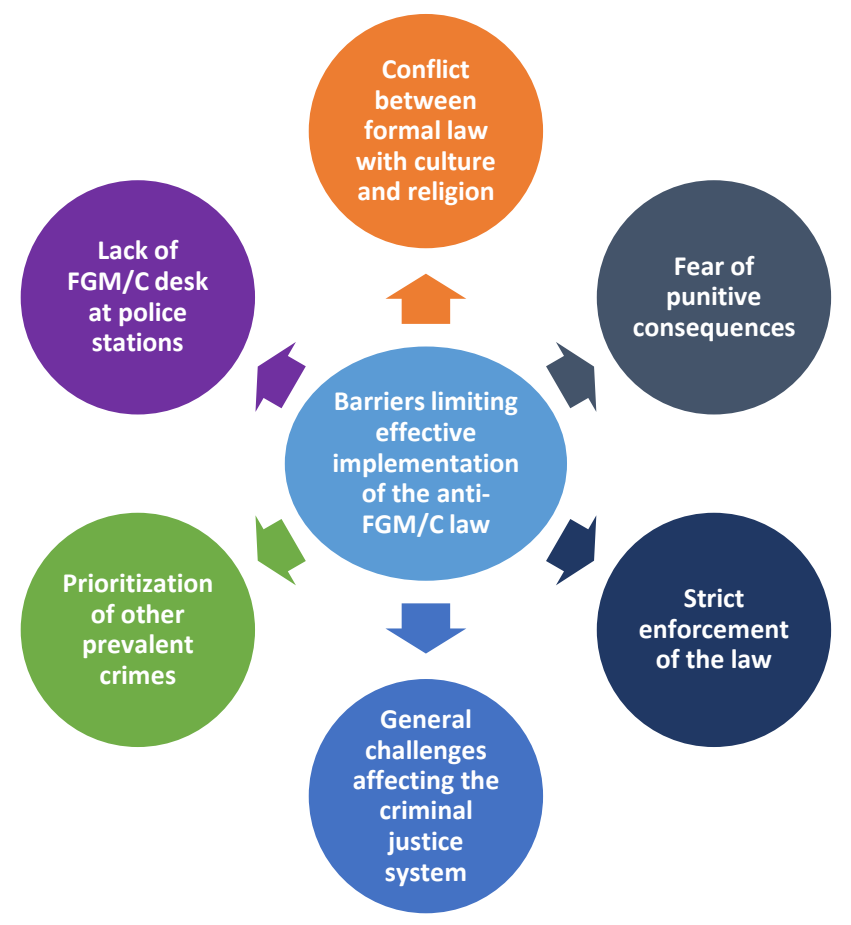

(Source: Meroka-Mutua, Mwanga, and Olungah 2020)

Details on the key barriers are as follows:

3.1.1 The formal law conflicts with culture and religion. Culture and religion influence the behaviour of individuals within communities, and where a conflict occurs, individuals do not always choose to obey the formal law over cultural or religious beliefs.

3.1.2 Fear of punitive consequences leads to lower reporting of FGM/C. In Kenya, the law drives the practice underground and individuals feel they cannot freely make reports about FGM/C occurring in their families and communities to government authorities, for fear of punishment.

3.1.3 Strict enforcement of Kenya's FGM/C law, particularly in light of the mandatory minimum sentences, has negative social impacts. For example, imprisonment of parents convicted for offences under the Act can leave children without primary carers, and in extreme cases, children would then have to be placed under the care of the State. Law enforcement officials face the dilemma of either enforcing the law and risk negative social impacts, or failing to enforce the law allowing FGM/C to continue.

3.1.4 The general challenges affecting the criminal justice system in Kenya make it difficult for the anti-FGM/C law to be properly implemented and enforced. In rural FGM/C hotspots, law enforcement officials travel long distances between police stations, scenes of crime and court stations. Witnesses face similar challenges and often lack the resources to attend court sessions. 
This affects the way investigations are conducted, how evidence is collected, and ultimately how prosecutions are carried out.

3.1.5 Other crimes that tend to be prevalent tend to be prioritised at the expense of FGM/C. This happens more often under circumstances where there are limited financial and human resources. For example, crimes such as cattle rustling and illegal brewing of traditional alcohol routinely receive more attention from law enforcement officials as opposed to FGM/C.

3.1.6 The police do not have a specific desk that deals with cases of FGM/C. While there exists a special desk within the Office of the Director of Public Prosecutions dedicated to the prosecution of FGM/C-related offences, this is not the case at police stations. Although FGM/C cases may be handled by the Gender Desk in some stations, there is a need for more coordination among the various levels and institutions tasked with the enforcement of the law.

\subsection{Recommendations for using the law more effectively towards abandonment}

The recommendations that follow were proposed for consideration through consultations with representatives from the Office of the Chief Justice of the Republic of Kenya, the Office of the Director of Public Prosecutions, the Kenya Law Reform Commission, the Law Society of Kenya, UNICEF-Kenya, and the Anti-FGM Board. They do not reflect variations or levels in key actor agreements/disagreements to each, nor do they reflect the review of the Council or any consortium partner.

Figure 4. What to consider for effective implementation of the anti-FGM/C law

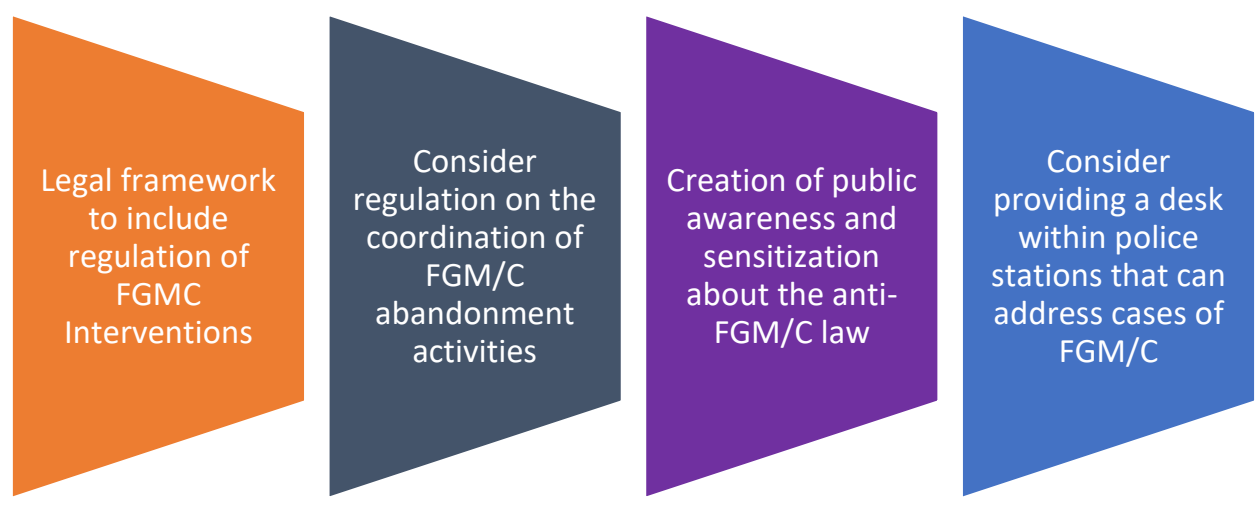

(Source: Meroka-Mutua, Mwanga, and Olungah 2020).

Figure 4 highlights key recommendations to be considered for effective implementation of the Anti$\mathrm{FGM} / \mathrm{C}$ law.

\subsubsection{Kenya's legal framework aimed at eliminating FGM/C relies mainly on criminalisation} of the practice. Consideration should also be given to the regulatory potential of the law. Regulation in this context is used to refer to legal provisions that address other $\mathrm{FGM} / \mathrm{C}$ interventions, such as community health services, education, and grassroots activities by community and religious leaders. In Kenya, the 2011 Prohibition of FGM Act incorporates both criminalisation and regulation, but criminalisation receives greater focus. The law should include regulations that recognise religious and cultural leaders, community health workers (CHWs), and even teachers as key actors in abandonment efforts and provide structures for how these groups may work within existing institutional frameworks. The establishment of these regulations could take a bottom-up approach, adhering to the constitutional principle of public participation, to enable community buy in. 
3.2.2 The law should include within it the regulation and coordination of key activities aimed at the abandonment of FGM/C. While the 2011 Prohibition of FGM Act provides for other measures that the government shall take to address $F G M / C$, including the provision of support measures to survivors of $F G M / C$ and public education and sensitization on the dangers of $F G M / C$, there are no clear provisions as to the implementation of these activities and the institutions that are required to carry them out. Implementation of the law may be strengthened by providing for the regulation and coordination of all anti-FGM/C activities. In developing these regulations, the roles and functions of county governments should be clearly stipulated.

3.2.3 Creation of public awareness and sensitisation about the anti-FGM/C law should not simply focus on the punitive sanctions under the law. Instead, awareness creation about the law should use positive messaging to encourage individuals to follow the law for their good and that of the community. Such positive messaging should be developed and disseminated in collaboration with key actors in the communities, including religious and cultural leaders, as well as community health workers.

3.2.4 Implementation of the anti-FGM/C law could be strengthened by providing for a desk within police stations in FGM/C hotspots that can address cases of FGM/C. This could either be a new desk dedicated to $\mathrm{FGM} / \mathrm{C}$ or the capacity of existing gender desks could be strengthened to manage such cases. This desk could work in coordination with the special desk within the Office of the Director of Public Prosecutions and the Anti-FGM Board.

\section{Changes in Practise and Social Norms Associated with FGM/C}

Figure 5. Changes in FGM/C practises

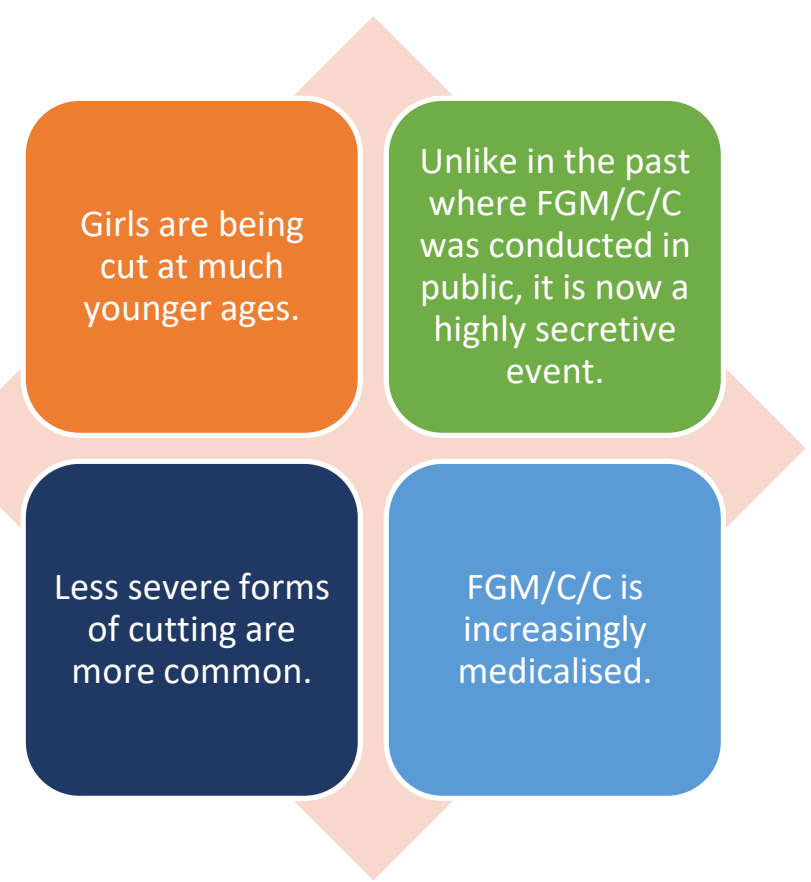

(Source: Matanda et al. 2018; Kimani et al. 2018)

Many $\mathrm{FGM} / \mathrm{C}$ interventions including legal reforms, community-based programmes such as alternative rites of passage, community dialogues, and girls' rescue programmes, and indirect change efforts such as promotion of girls' education have been implemented in Kenya (Matanda 
et al. 2018). Given implementation of these activities, it is important that we begin providing answers to the key question: to what extent have interventions facilitated $\mathrm{FGM} / \mathrm{C}$ abandonment? Findings from the Evidence to End FGM/C Research Programme conducted among the Maasai and Kisii have shown that the practice is evolving (Figure 5) - being done in secret, shifting towards lesser severe cuts at younger ages, and increasingly performed by health professionals rather than traditional cutters (Matanda et al. 2018; Kimani and Kabiru 2018). These changes have occurred in response to laws banning the practice and awareness-raising campaigns focused on the health risks of FGM/C. Some families are learning to manage health risks and avoid legal consequences while preserving cultural values (Matanda et al. 2018; Kimani and Kabiru 2018).

Regarding changes in norms associated with FGM/C, evidence shows that the practice is rooted in gender inequality, ethnic identity, and social and religious affiliations (Matanda et al. 2018; Kimani and Kabiru 2018). For example, as shown in Figure 6, among the Maasai in Narok County and the Abagusii in Kisii County FGM/C is believed to promote sexual restraint, thus upholding morality in the community and fidelity in marriage. However, reduced sexual desire is believed to lead to marital instability as men perceive sex with cut women less sexually satisfying than with uncut women. Additionally, $\mathrm{FGM} / \mathrm{C}$ is widely believed to heighten a girl's chances of being married early, but there is ambivalence regarding FGM/C being a prerequisite for marriage as it is increasingly acceptable to marry an uncut woman. In Narok and Kisii, FGM/C confers ethnic identity, with women who do not conform sometimes ostracised within their social networks. In Kisii, cut women were respected by community members and considered respectful of others, while in Narok it was the opposite, where cut girls considered themselves equals to senior community members and hence disrespectful (Matanda et al. 2018).

Figure 6. Changes in social norms associated with FGM/C
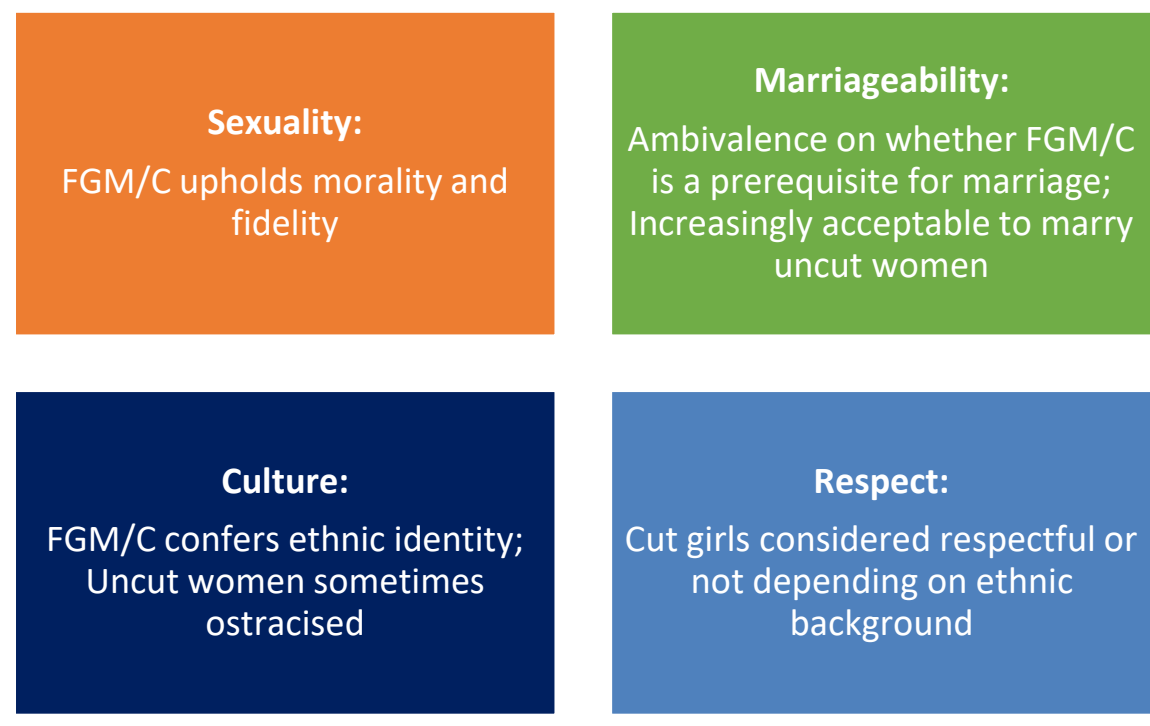

(Source: Matanda et al. 2018)

4.1 Policy and programmatic recommendations for changes in FGM/C practise and social norms

What we have learned from the observed changes in practise and norms is that even though the prevalence of $\mathrm{FGM} / \mathrm{C}$ has remained high in selected communities, there are substantial changes happening in the community. How then can policymakers and programme implementers leverage these changes to promote total abandonment? The following should be considered:

4.1.1 There is need for creation of platforms that support deliberative processes at both the individual and community level calling into question the perceived benefits of $F G M / C$. The healthcare system has an important role to play especially in addressing medicalisation. 
Interventions including provision of in-service and pre-service training on $\mathrm{FGM} / \mathrm{C}$ and strengthening the capacity of the healthcare system to prevent and monitor FGM/C incidents and manage FGM/C related health issues, human rights, and bodily integrity should be implemented. This requires a multi-sector approach in addressing these issues. The use of social media campaigns is a viable platform given its growth and popularity in Kenya.

4.1.2 Promote community discussion of women and men's roles in decisions on FGM/C. Critical reflection and community dialogue on gender norms that determine culturally acceptable notions should be included in programmes working towards abandonment. The shift in norms where men prefer uncut women calls for involvement of men and boys in the implementation of $\mathrm{FGM} / \mathrm{C}$ interventions with clear roles that are sensitive to cultural context.

\section{FGM/C Practise: The Key Health-Sector Learnings for Policy and Practise}

The negative impact of $\mathrm{FGM} / \mathrm{C}$ on health and well-being calls for a coordinated health-sector response to prevention and management of the practice. The health sector is better positioned to lead the $\mathrm{FGM} / \mathrm{C}$ response because of its primary role in disease prevention, health promotion, and provision of curative services. The sector's response to FGM/C is anchored on the nation-wide and sector-specific legal/policy frameworks and guidelines adopted from global and regional instruments as well as the WHO. Despite the strategic position occupied by the health sector, its critical role, and the existence of a favourable legal/policy environment that should direct a robust health system's response to $\mathrm{FGM} / \mathrm{C}$, the practice has persisted, while medicalisation is on the increase. In response to the noted challenges, the Evidence to End FGM/C Research Programme conducted studies to investigate the extent of medicalisation of $F G M / C$ in a number of African settings (Egypt, Sudan, Nigeria, Somaliland) including selected communities in Kenya (Abagusii, Kuria, and Somali) (Kimani and Kabiru 2018). Moreover, a diagnostic assessment of the health system's response to $\mathrm{FGM} / \mathrm{C}$ prevention and management in Kenya was conducted to understand the preparedness of the health system (Kimani and Okondo 2020).

\subsection{Key findings on medicalisation of FGM/C}

\subsubsection{Communities are increasingly adopting medicalised FGM/C}

Community members belonging to the Abagusii and Somali ethnicities in Kenya have increasingly adopted medicalisation of $\mathrm{FGM} / \mathrm{C}$. The procedure is performed at home or in private clinics during off-hours on long holidays. This provided a perfect environment to perpetuate the practice in secret for fear of legal sanctions. The motivation for medicalisation among health workers included the perceived desire to mitigate health complications and financial compensation. Additionally, the health workers belong to communities that practice FGM/C, therefore faced social pressure to perform $\mathrm{FGM} / \mathrm{C}$ or face being ostracised, which may affect performance of their important healthcare roles. The accompanying elements of medicalisation noted were less severe cutting and younger age at cutting. For example, among the Somalis where medicalisation is common, Sunna-a less severe form of cutting - is commonly practiced. Although from the community view, Sunna is not perceived to be a form of FGM/C, diverse descriptions give an indication that Sunna is FGM/C that could span from type IV, I, or even type II. Furthermore, apart from religion, medicalisation was associated with increased education, awareness of health complications, and perceived sexual advantage to men and women. However, families in the Kuria and West Pokot communities have not adopted medicalisation. The stability of FGM/C in these communities was associated with the practice serving as a rite of passage and hence the higher age at cutting. 


\subsubsection{Recommendations for policy and practise to address medicalisation}

Figure 7 highlights strategies relevant to policy and practise in addressing medicalisation.

First, there is need for creation of awareness on FGM/C-related complications and illegality of medicalisation. In hotspot areas for medicalisation, interventions targeting parents, birth attendants, health facilities, and healthcare providers are critical. These interventions should include creation of awareness on the health impacts of $\mathrm{FGM} / \mathrm{C}$, illegality of the practice, clarification that medicalisation is associated with health complications, as well as violation of women's and girls' rights. Such programmes should involve religious leaders, the council of elders, and other custodians of culture to increase uptake and trigger norm shift away from support for continuation of $\mathrm{FGM} / \mathrm{C}$.

Figure 7. Strategies relevant to policy and practise for addressing medicalisation

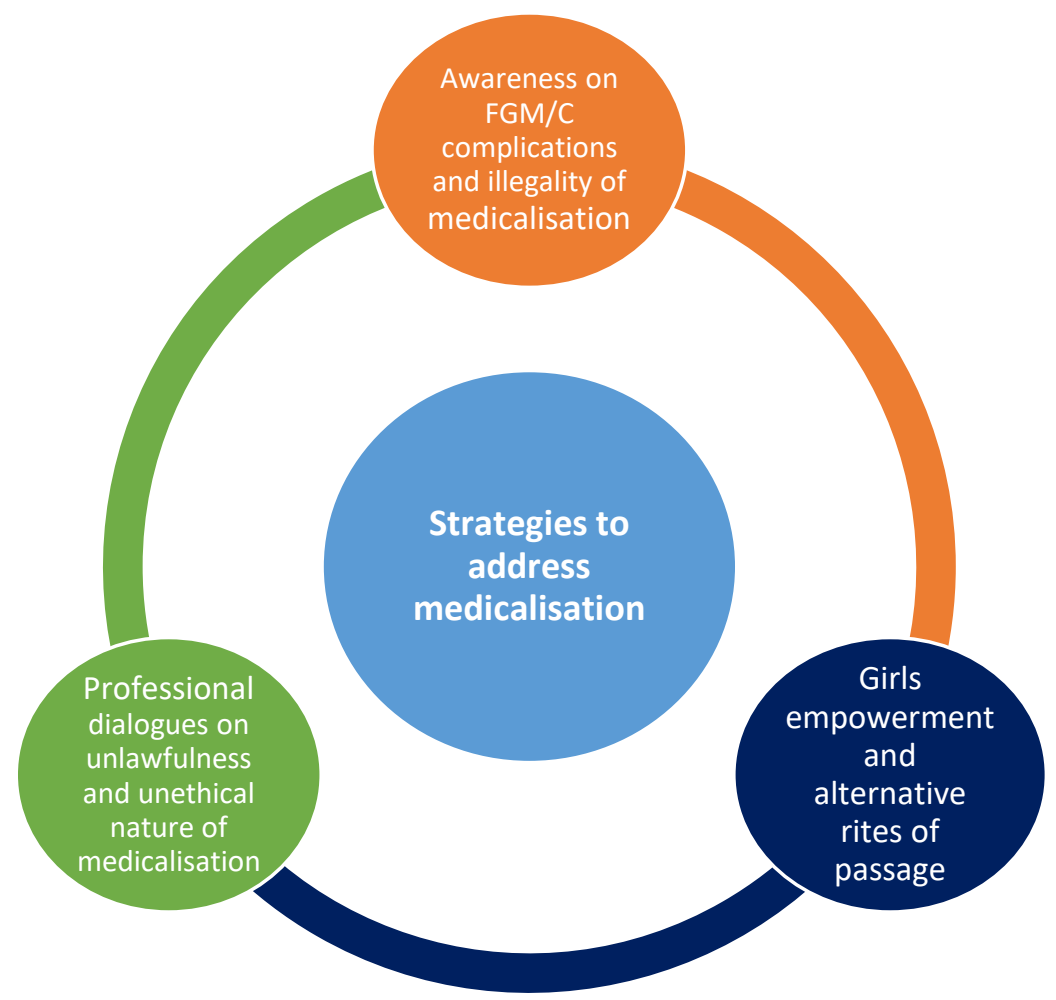

(Source: Kimani et al. 2018; Kimani et al. 2020)

Second is the implementation of girls' empowerment and alternative rites of passage. In communities where girls are cut at a late age, interventions should involve girl empowerment through promotion of formal education, life-skills training, alternative rites of passages as well as school-related interventions articulated in the national school health policy (MOE 2018). Furthermore, specific interventions should target girls in hard-to-reach and remote areas implemented through communities, county governments, NGOs, and CBOs.

Third, is the need for conducting professional dialogues and mapping social networks. Professional dialogues should include consideration of health workers' awareness about unlawfulness and unethical nature of medicalisation. The social networks should be determined for providers from the affected communities to address their own concerns and how best to deal with social pressures that force them to medicalise. These interventions should be implemented through involvement of professional regulatory bodies, associations, and societies as a 
mechanism of reinvigorating professional self-regulation, re-emphasis on the "Do No Harm" principle, and development of disciplinary procedures.

\subsection{Key findings relevant to prevention and management of FGM/C-related complications}

\subsubsection{Laws and policies addressing response to FGM/C exist but there is limited knowledge} about their contents: The health sector is guided by the nationwide and sector-specific FGM/Crelated legal/policy documents that address prevention and management of $F G M / C$. These include: the Constitution of Kenya that guarantees fundamental rights; the Prohibition of FGM/C Act of 2011 that defines offences and punishments/fines related to FGM/C; the Children Act of 2001 that protects and safeguards girls below 18 years; and the Protection against Domestic Violence Act of 2015 that categorises FGM/C as a form of violence. Health-sector-specific legislation, notably the Medical Practitioners and Dentist Act, Nurses Act, and Clinical Officers Act, are not explicit on response to FGM/C (Kimani and Okondo 2020). Additionally, there are specific health-sector policies that address FGM/C prevention and management including the National Adolescent Sexual and Reproductive Health and National School Health policies. There was, however, limited knowledge across the stakeholders of their contents regarding FGM/C prevention and management that was attributed to lack of dissemination.

5.2.2 Limited skills to respond to FGM/C-related complications: Many women and girls sought treatment for FGM/C-related complications, however, few received full care in the first health facility (Kimani and Okondo 2020). This means that clients had to navigate extra distance and hours, and incur extra cost in seeking services which may deter health-seeking (Kimani et al. 2020). Indeed, most of the women and girls were referred to higher-level facilities because of providers' limited skills at lower level facilities. Furthermore, health providers had challenges performing deinfibulation to address FGM/C-related birth complications prompting bilateral and "generous" episiotomies and caesarean sections. Surprisingly, there were no interventions implemented to mitigate sexual and psychological complications (Kimani and Okondo 2020).

\subsubsection{Lack of FGM/C data capture and documentation in health service delivery system:} Consistently throughout the health facilities and in-service delivery points (antenatal, postnatal, family planning), there were no data on women/girls with FGM/C and those with practise-related complications. Although women/girls presented themselves to the facilities with FGM/C-related bleeding and infections, among other complications, there were no data from health-facility assessments to support this claim -an indication of lack of adequate recordkeeping (Kimani and Okondo 2020).

5.2.4 Few health-related FGM/C prevention interventions: The health infrastructure and human capacity are adequate to conduct preventive interventions for priority programmes at the county level, but minimal FGM/C prevention activities were implemented. Counselling of clients with $\mathrm{FGM} / \mathrm{C}$, and creation of awareness of complications, and of the need to protect daughters against FGM/C were rarely implemented. Whereas counties boast of infrastructure including health facilities, schools, vernacular radio stations, and community health volunteers that could be helpful in FGM/C-related prevention, limited FGM/C-related activities leverage these resources. Furthermore, health facility-linked interventions such as health talks and community-based activities including school-based programmes and outreach work were reportedly hindered by lack of guidelines; protocols; information, education, and communication (IEC) tools, limited capacity of health workers; and lack of funding (Kimani and Okondo 2020).

5.2.5 Women/girls still experience FGM/C-related complications but rarely receive care and services: Women and girls sought care for a wide range of complications including immediate physical, birth, sexual, and psychological complications. Most of the birth complications included tears on the external genitalia, and postpartum haemorrhage that required episiotomies, and 
caesarean section. Long-term complications included fistulas, scars, and keloids, while psychological impacts included psychological trauma and stigmatisation. Also noted were FGM/Crelated sexual complications characterised by difficulty in penetration, painful intercourse, and lack of enjoyment. There was no evidence on interventions offered to mitigate psychological and sexual complications (Kimani and Okondo 2020).

5.3 Recommendations for policy and practise regarding prevention and management of FGM/Crelated complications

Key considerations for policy and practise in preventing FGM/C and managing FGM/C related complications are summarised in Figure 8. They include:

5.3.1 Targeted training of healthcare providers: Utilizing mapping data of hotspot counties with their corresponding types of $\mathrm{FGM} / \mathrm{C}$ should provide an indication of where targeted training for FGM/C interventions is required. This approach is already being implemented by UNFPA's funded healthcare provider training programmes in hotspot counties and thus need to be scaled up. Training interventions should include identification, management of FGM/C and referral pathway for women and girls with complications. Implementation of targeted training workshops on specific skills like deinfibulation for birth complications associated with infibulation in counties like West Pokot and Garissa is critical. In addition, trainings to empower professionals address FGM/Crelated sexual and psychological complications is paramount. This should include counselling and psychosocial support interventions as well as FGM/C prevention approaches (Kimani et al. 2018).

Figure 8. Considerations for policy and practise regarding prevention and Management of FGM/C related complications
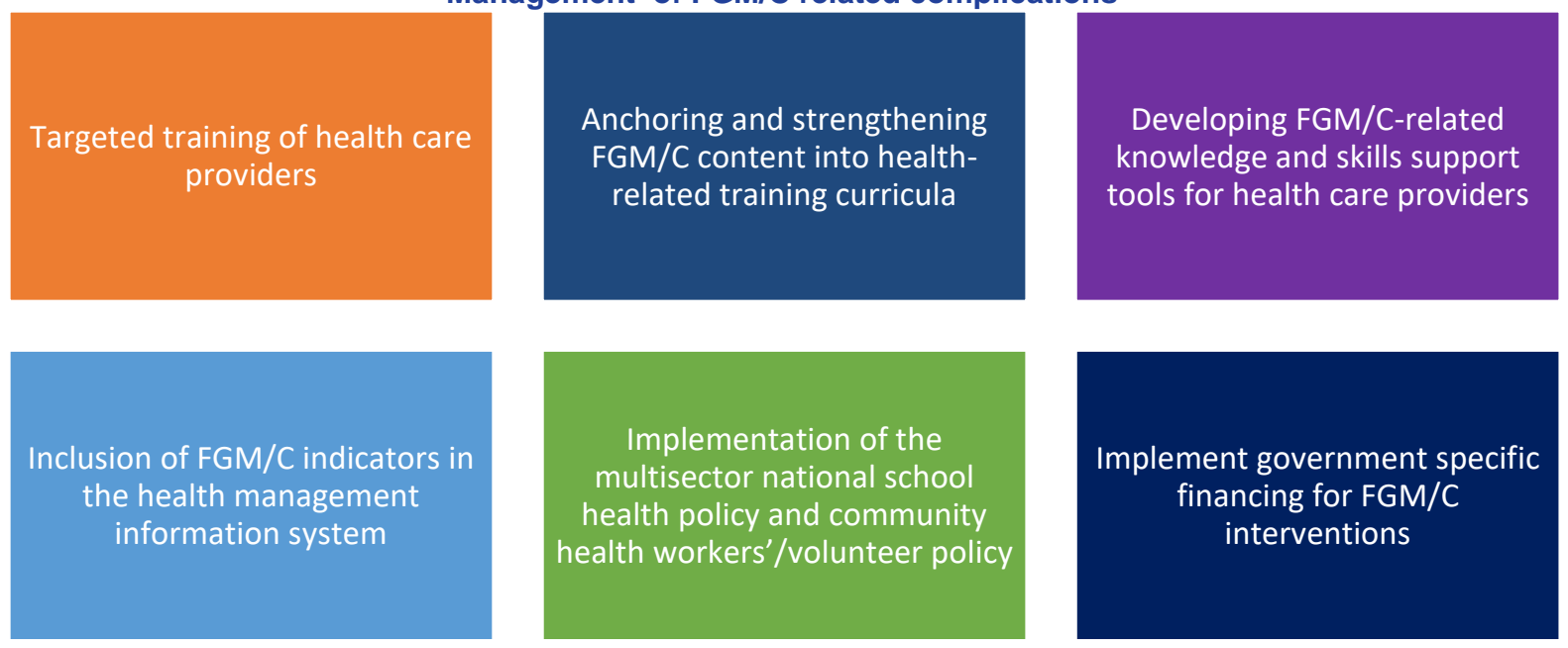

(Source: Kimani et al. 2020)

5.3.2 Anchoring and strengthening FGM/C content into health-related training curricula: To build capacity for health workers to respond to prevention and FGM/C-related complications right from pre-service training. Similarly, for qualified practising health professionals, training modules should be developed for inclusion in continuous professional development to address $\mathrm{FGM} / \mathrm{C}$ related knowledge and skills gaps. Exploitation of innovative strategies such as electronic tools (etool) should be explored to address staff shortages (Kimani et al. 2018).

\subsubsection{Develop FGM/C-related knowledge and skills support tools for healthcare providers:}

To ensure high-quality care for women/girls with FGM/C-related complications (e.g., birth, gynaecological, and sexual), there is a need for building the competency and skills of healthcare providers and equip them with proper support tools. To begin, a review of the 2007 Ministry of Health's manual on FGM/C management and prevention (MOH 2007) should be conducted 
incorporating recommendations from the latest WHO handbook and manuals (WHO 2018). Development of innovative ways for training health workers in high-prevalence FGM/C counties should also be prioritised. Additionally, there is need for sensitisation of providers through provision of knowledge tools at the service delivery points.

\subsubsection{Ensure inclusion of FGM/C indicators in the health management information system} (HMIS) and development of FGM/C data capture tools: Currently FGM/C-related data, for example number of women delivering with $\mathrm{FGM} / \mathrm{C}$ and common complications women present with at health facilities are not captured. Importantly, FGM/C indicators that would enable collection of $\mathrm{FGM} / \mathrm{C}$ data have not been anchored in the HMIS. There have been fruitful discussions with the Ministry of Health culminating in development of FGM/C indicators for inclusion in the HMIS but data str not input at service delivery points. This calls for training of healthcare providers on FGM/C issues, identification of types of FGM/C and related complications, data acquisition, dissemination, and use of the data-collection tools. The development of the FGM/C data capture tool can leverage the post-rape care (PRC) form used in gender-based violence case management. This may promote integration of $\mathrm{FGM} / \mathrm{C}$ into existing health programmes, monitoring, and evaluation pathways (Kimani et al. 2018; Ministry of Public Health 2009).

5.3.5 Implement the multisector national school health policy and community health worker/volunteer policy: A national school health policy envisages collaboration between the Ministries of Education and Health on matters of FGM/C interventions among schoolgoing children (MOE 2018). Actualisation of this policy should address girls' empowerment and school-based FGM/C interventions, and enhance collaboration between the health sector, education, and the community. Additionally, with universal healthcare gaining momentum, the policy's success could be promoted through community health workers/volunteers. A policy that exists for this cadre of community members, thus leveraging them, has potential for success in FGM/C prevention interventions. County governments can identify, train, and engage $\mathrm{CHWs} /$ volunteers to execute FGM/C-related interventional activities at the community level.

5.3.6 Implement government-specific financing for FGM/C interventions: Funding of FGM/C interventions has been limited even in the high-prevalence counties. Raising awareness and linking $\mathrm{FGM} / \mathrm{C}$ to socioeconomic and health impacts/challenges could enlighten and stimulate stakeholders, especially policymakers at the county level, to take actions including financing for the FGM/C-related interventions. Integration and cost analyses to examine the financial implication of integration of $\mathrm{FGM} / \mathrm{C}$ interventions into existing health services and education services are needed. Advocacy for gender-responsive budgeting is also needed to hold the national and county governments accountable for investing in the prevention and management of FGM/C.

\section{The Continuing Need for FGM/C Data: What Do We Need to Know and Why Do We Need to Know It?}

6.1 Data collected as evidence for the need for abandonment, and progress towards abandonment

To accelerate FGM/C abandonment, all FGM/C stakeholders should access and use high-quality data to guide interventions and measure change. Recently, there have been a marked increase in the amount of systematically collected data on FGM/C. These data has been collected through a range of methods, including medical case records, small-scale surveys, and larger national surveys like the Kenya Demographic and Health Surveys (KDHS) and Multiple Cluster Indicator Surveys (MICS). These data sources, especially KDHS and MICS, have greatly increased understanding about the practice and have been used extensively to advocate for abandonment 
of FGM/C (Shell-Duncan, Naik and Feldman-Jacobs 2016; Kandala et al. 2017; Kandala et al. 2020a; Kandala et al. 2020b).

Aside from these national datasets, Kenya continues to face challenges with regard to data quality, processing, and usage. Some of the areas we provide insights for and worth noting include:

- Many local organisations implementing interventions lack the capacity to systematically collect quality monitoring and evaluation data that can be shared and used to inform programmes and policy. This therefore means that interventions are implemented without a clear pathway of change and are rarely informed by evidence. Emphasis on developing a Theory of Change by local organisations right from the planning and structuring the evaluation around it would make a huge difference (Population Council 2020).

- There is lack of real-time data on FGM/C as national surveys such as KDHS and MICS are often implemented only every five years. Real-time data are important because FGM/C is driven by social norms, attitudes, and beliefs that keep changing and hence the need to tailor interventions based on the most current changes observed. Given the challenge of FGM/C being practised in secret, what may be feasible is capturing as much data through HMIS for women seeking care and who have FGM/C-related complications (Kimani and Okondo 2020). In addition, this may involve collection of $F G M / C$ data at health facilities or continuous gathering and use of data on programme implementation, with the aim of ensuring programmes are proceeding satisfactorily or making adjustments. It may also involve a more comprehensive assessment of programmes undertaken at discrete points in time focusing on the longer-term outcomes and impacts of the programmes. These data may be focused on local initiatives as well as measuring effectiveness of interventions at the national and regional levels (Population Council 2020).

- Inability to disaggregate national-level data. Most national-level surveys (MICS, DHS) are not statistically powered to disaggregate data to more refined analysis at lower units of administration. To adequately design, implement, and monitor interventions at the county level, or lower, locally collected data are needed.

\subsection{FGM/C data: Prevalence for 15-49-year-olds and 0-14-year-olds}

National household surveys such as DHS and MICS collect data on various health conditions including a module on FGM/C. Analysis of questions in the FGM/C module across respondents' socioeconomic and demographic characteristics have been used to study trends in FGM/C (Kandala et al. 2017; Kandala et al. 2020a; Kandala et al. 2020b). The analysis commonly focusses on the prevalence for women aged 15-49 years over a period of time. However, focussing on prevalence among women aged 15-49 years provides limited information about recent trends as most of the women in this age group experienced FGM/C many years ago. The Evidence to End FGM/C expanded on this approach by analysing data in five-year age cohorts (Shell-Duncan, Gathar, and Moore 2017) across multiple rounds of the DHS. The age cohort analysis can be helpful in documenting when changes in FGM/C prevalence happened and in which demographic groups. For example, Kenya experienced a steady, long-term decline in rates of FGM/C that began in the early 1980s but the rate of decline did not occur evenly among women across all ethnic groups. Rates have remained substantially high among women of Somali ethnicity. Rates are equally high in older cohorts among the Maasai and Kisii women but have declined among younger cohorts (Shell-Duncan, Gathar, and Moore 2017). 
Figure 9. Person who performed FGM/C among women and daughters

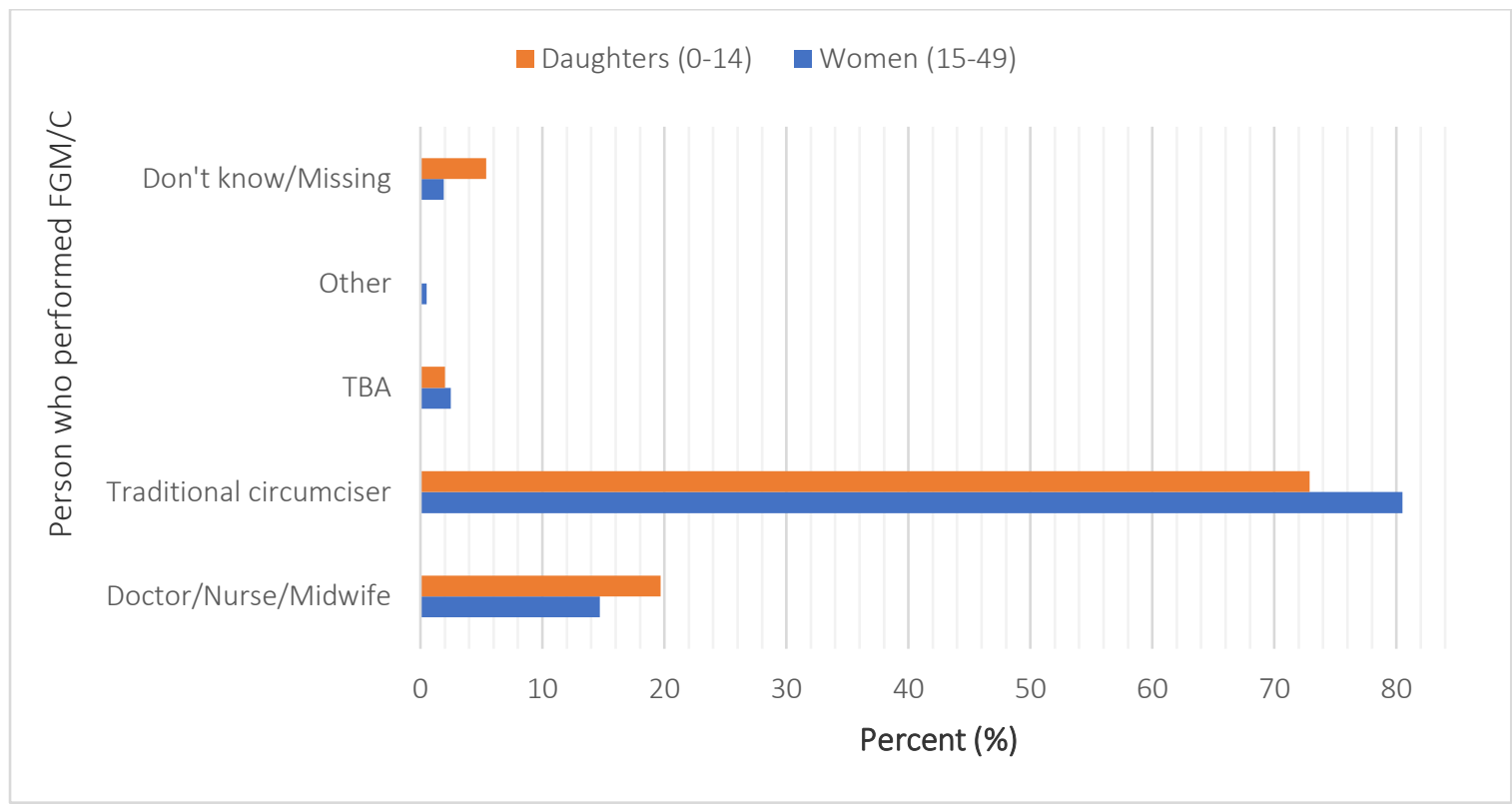

(Source: Shell-Duncan, Gathara, and Moore 2017)

FGM/C may persist or undergo changes from generation to generation. It is therefore important that we understand mothers' and daughters' differences or similarities regarding their $\mathrm{FGM} / \mathrm{C}$ status, attitudes, and practises towards FGM/C. For example, by examining data on the person who performed FGM/C for mothers versus for daughters (Figure 9), evidence shows that the rate of medicalisation at a national level has increased for girls $0-14$ years $(20 \%)$ as compared to women 15-49 years (15\%) (Shell-Duncan, Gathar, and Moore 2017). This juxtaposition is important because such changes could be driven by beliefs that it is safer to conduct FGM/C under sterile conditions by trained health professionals; and yet this shift is attempting to modernise and legitimise an illegal practise (Kimani and Shell-Duncan 2018).

Detailed analysis of data for daughters aged $0-14$ years can equally provide insightful information, especially on the possible influence of the immediate social environment and interventions. Nonetheless, the analysis and interpretation of data on daughters is complex as not all girls have reached their final cutting status. Data for girls aged 0-14 years is therefore affected by "censored observations"-cases in which a girl may still be at risk of being cut in the future. Thus, a statistical technique known as survival analysis is usually used to ensure that such data can be used reliably (Kandala et al. 2017; Kandala et al. 2020a; Kandala et al. 2020b).

\subsection{Mapping of hotspot areas and its relevance for policy and programming}

The KDHS data contain geographic information relating to locations where mothers and daughters who have undergone $\mathrm{FGM} / \mathrm{C}$ reside, together with their sociodemographic information. Analysis of this information can provide a powerful means of studying how community factors (e.g., area of residence), social norms (e.g., attitude, beliefs, and practises concerning FGM/C), and individual factors (e.g., educational attainment, socioeconomic status, and ethnicity) influence FGM/C practise. The Evidence to End FGM/C Research Programme used advanced statistical methods (Bayesian multivariate spatial analysis) to conduct within-country analyses using KDHS data (Kandala et al. 2017). Survival analysis was used to predict the likelihood that a girl aged 0-14 years would be cut based on her geographical location and sociodemographic characteristics. The analysis examined how FGM/C prevalence varied over time and by geographic location through mapping of prevalence at the national level and in the 47 counties (Figure 10). 
Figure 10. Predicted FGM/C prevalence among girls 0-14 years, KDHS 1998 to 2014

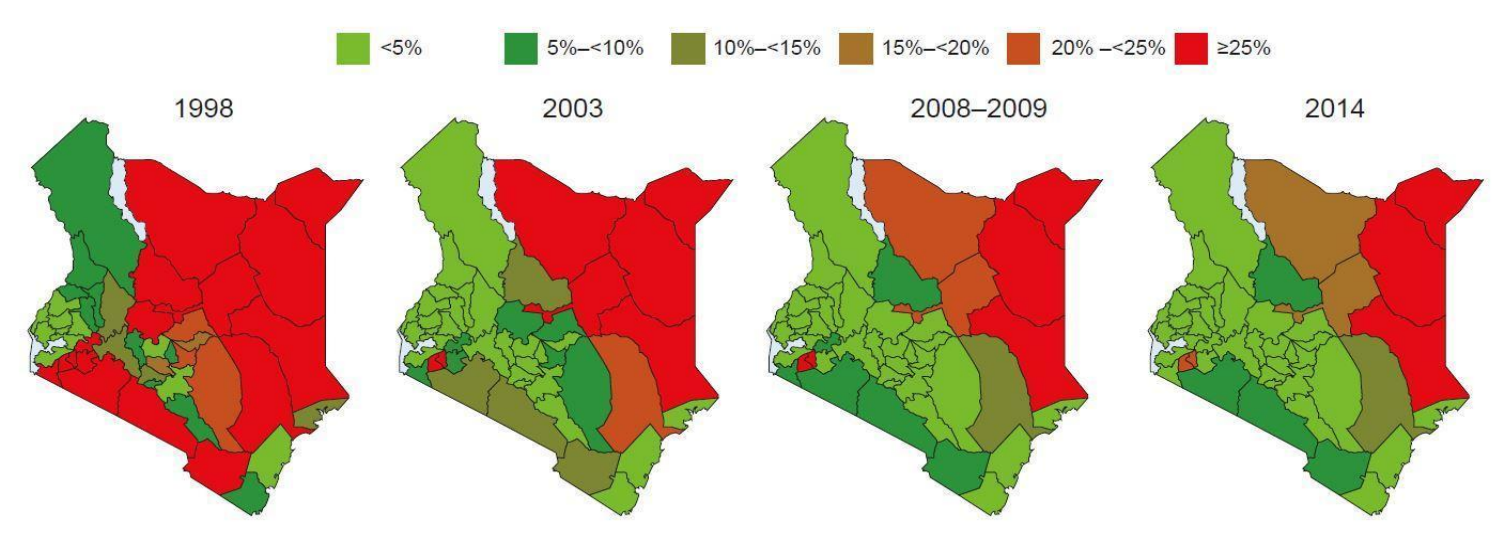

(Source: Kandala et al. 2017)

The focus on data for girls aged 0-14 years was important because patterns of FGM/C prevalence among the youngest age group reflect the most recent changes to the practise. Key findings identified Mandera (47\%), Wajir (46\%), Garissa (36\%), Kisii (24\%), and Nyamira (23\%) as hotspot areas. Survival analysis showed that FGM/C occurred at much younger ages among daughters aged 0-14 years compared with their mothers. Additionally, data showed girls undergo the most severe forms of FGM/C (Type III) much earlier than other types; after girls were five years old, FGM/C occurred much earlier in urban areas than in rural areas; FGM/C occurred at younger ages in the North-Eastern region than in the Rift-Valley and Central regions; FGM/C occurred earlier for Muslim girls and among ethnic Somali girls; and girls belonging to mothers with no education and living in the poorest households were cut at much younger ages. Regarding the influence of social norms, daughters were more likely to be cut if they lived in communities where the majority of women were cut, a greater proportion of women supported the continuation of FGM/C, and more women believed that FGM/C was required by community and/or by religion (Kandala et al. 2017).

The mapping of hotspot areas and groups that require special attention is useful both for policy and programmatic interventions as highlighted below:

- In Kenya, the prevalence of FGM/C tends to be concentrated in certain locations and therefore considering only national-level data may conceal important local variations of the practice. This calls for subnational policies and initiatives to end FGM/C that leverage governance structures at the county level.

- Social norms are a key factor driving FGM/C in Kenya. Belonging to a certain ethnic group (e.g., Somali or Kisii), religious group (Muslim), and living in geographical areas where a bigger proportion of women have undergone FGM/C increases the likelihood of girls being cut. What this means is that individuals' decisions are likely to be shaped by common practises in the community where they live. Therefore, FGM/C is likely to persist because of shared expectations within social groups. Understanding these dynamics can help in designing abandonment initiatives that contribute to shifts in community norms.

- Policy and programmatic interventions should consider the age at which FGM/C occurs. Given that the age at cutting has declined in Kenya, behaviour-change interventions focused on the decisionmaking dynamics need to be informed by the local context rather than promoting alternative rites of passage that may not be relevant in certain settings. 
- Initiatives that aim to promote women's and children's rights through gender equality and women's empowerment are needed. This is critical especially in contexts where FGM/C prevalence is higher among girls whose mothers have no education and live in poverty.

\subsection{Challenges and opportunities with measuring the impact of $F G M / C$ programmes}

Kenya has experienced numerous efforts aimed at ending FGM/C. These include legal reforms, implementation of community-based interventions such as alternative rites of passage, community dialogues, girls rescue programmes, and promotion of girls' education. Even though implementation of these interventions has been intensified in the last decade, there is limited knowledge on what interventions are most effective and worthy of scale up. This can be attributed to the lack of a clear Theory of Change, weak methodological approaches, and inadequacies in the design of FGM/C interventions (Berg and Denison 2012). Significantly, many interventions are implemented by local organisations with inadequate resources to document and evaluate their activities. In addition, the complex nature of interventions aimed at addressing violence against women and girls, including $\mathrm{FGM} / \mathrm{C}$ makes it difficult to adequately document the implementation process and outcomes (Population Council 2020; Berg and Denison 2012).

Despite these challenges, evaluation of FGM/C interventions and monitoring of change is critical and should be prioritised by policymakers and programme implementers. This will enable sharing of knowledge on what works; lessons learned; and how to adapt, replicate, and scale up successful interventions.

Figure 11 summarises key issues that should be considered when conducting evaluation of $\mathrm{FGM} / \mathrm{C}$ interventions. These include:

- Objectives of the intervention and envisaged pathways towards outcomesindicators that will facilitate measurement of changes in behaviour and prevalence are key and should be considered at the outset;

- Potential other factors responsible for the observed changes-preferably through the use of experimental evaluation design, identification and documentation of other FGM/C activities within and near project sites, and reviewing history of FGM/C activities in project sites;

- Measurement of unplanned impacts of FGM/C interventions-to enable understanding of how interventions can influence people's lives beyond FGM/C. Document all events as they occur using qualitative research or conduct interviews retrospectively; and

- Ethical considerations - maximise potential benefits for individuals and society, minimise potential harms, respect the rights of individuals and groups, and protect persons with diminished autonomy. 


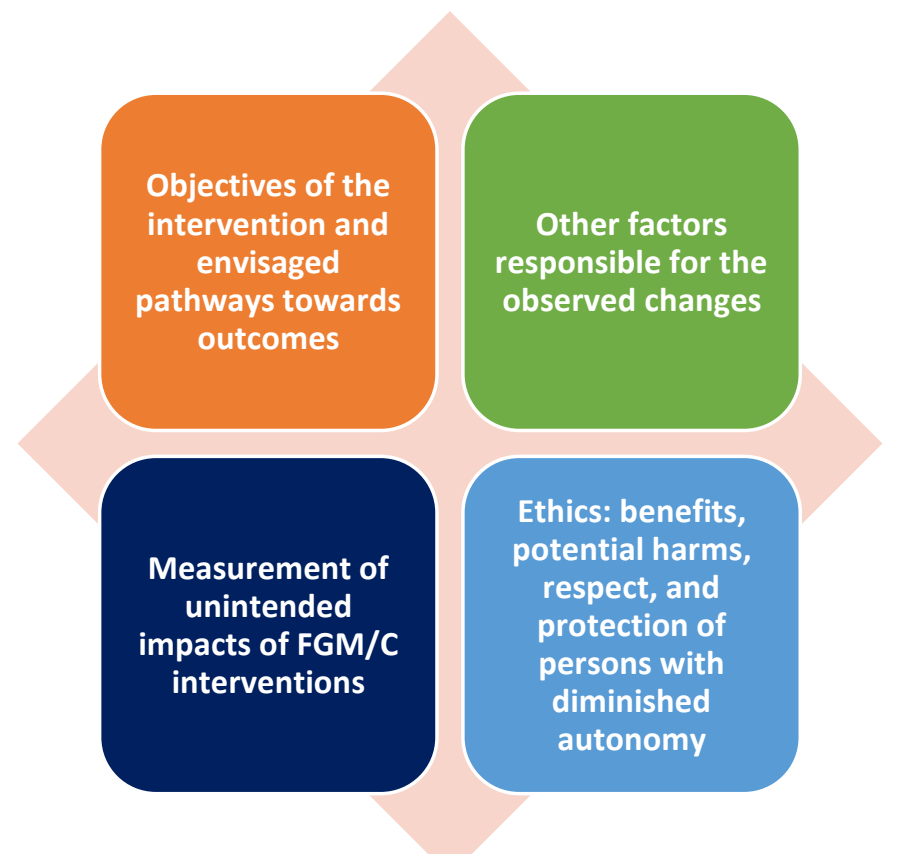

(Source: Guidance Note. Improving the rigor, relevance and utility of descriptions of FGM/C interventions and their evaluation. Population Council 2020)

\section{Recognising Structural Barriers and Responding with Structural Interventions}

The abandonment of $\mathrm{FGM} / \mathrm{C} / \mathrm{C}$ is often affected by structural barriers, which limit the extent to which $\mathrm{FGM} / \mathrm{C}$ interventions can be effective. Thus, there is a need to have structural interventions that are aimed at removing structural barriers. The projects under the Evidence to End FGM/C programme identified structural interventions which have had a positive impact in the fight against $\mathrm{FGM} / \mathrm{C}$ in Kenya.

\subsection{Education}

While FGM/C has been shown to curtail education opportunities for girls, there are now shifting trends in Kenya whereby the practice is performed on young girls before they commence school or on older women of childbearing age (6, Kandala et al. 2017). This means that more girls of schoolgoing age are now staying in school (Commonwealth Educatoin Hub 2016). Thus, there is an opportunity to educate girls and parents on the dangers of $\mathrm{FGM} / \mathrm{C}$ and to empower them to make the choice against the practice. The new, competency-based curriculum offers opportunities through which schoolgoing children can be taught about FGM/C and its harmful consequences. These opportunities should be leveraged and used effectively. Importantly, since FGM/C is driven by social norms, attitudes, and beliefs, involvement of the community where these girls live will be key (Cislaghi and Heise 2018; Cislaghi, Gillespie, and Mackie 2016). Further, implementation of the training curriculum for healthcare professionals on the management of $F G M / C$ that has been rolled out needs to be followed through. This will ensure that the health complications related to the practice are effectively managed, not just at high-level facilities but across facilities closer to those affected. 


\subsection{Economic status}

One of the reasons for the practice of $F G M / C$ is to increase the marriageability of women (Mackie and LeJeune 2009). In turn, marriage is seen as an important prospect for women, as it is a means through which women can access economic resources. In certain communities in Kenya, we have observed that FGM/C heightens a girl's chances of being married early but community members are increasingly accepting marriages that include uncut women (Matanda et al. 2018). However, in communities where access to resources and wealth is disproportionately enjoyed by men, it is difficult for women to make independent decisions that go against established norms that disadvantage women (Abuya 2020; Gruenbaum 2001; Gruenbaum 2005; Mose 2008). Thus, women who are not economically empowered, and who do not have other means of accessing economic resources other than through marriage, will be more likely to undergo the practice, and they will also be more likely to allow their daughters to undergo the practice (Abuya 2020: Mose 2008; OHCHR et al. 2008). Through economic empowerment, women are able to access economic resources via employment and entrepreneurship. This will narrow the gender inequality experienced in many settings in Kenya where FGM/C is prevalent. Consequently, factors that facilitate $\mathrm{FGM} / \mathrm{C}$ such as early marriage will cease to be the only means through which such women can access economic resources. It is expected that economically empowered women will more likely make the choice against FGM/C, particularly for their daughters. They may also prefer to educate their daughters to the highest level possible to ensure that their daughters are also economically empowered. It is therefore important to link intervention activities geared towards the abandonment of FGM/C to women's economic empowerment initiatives (Cislaghi and Heise 2018).

\section{Reflections on Emerging Debates Around FGM/C in Kenya}

\subsection{Interventions: The FGM/C law}

Kenya's FGM/C law has been the subject of protests by women in practising communities. We have already seen that some of the barriers to the enforcement and implementation of the 2011 Prohibition of FGM Act are the conflict between this law and other semi-autonomous fields that influence the choices people make. Currently, a constitutional petition has been brought before the courts, challenging the constitutionality of the Act.

The basis of this constitutional petition is that the total prohibition of $\mathrm{FGM} / \mathrm{C}$, even for women who might voluntarily consent to the practice, interferes with women's enjoyment of the right to culture under Article 44 of the Constitution and the freedom of conscience, religion, belief, or opinion under Article 32 of the Constitution. This constitutional petition therefore raises important questions concerning how the issue of conflicting rights should be treated under the law. The courts have an opportunity to develop the jurisprudence in this area and to offer guidance about how the rights of women and girls at risk of $\mathrm{FGM} / \mathrm{C}$ should be safeguarded, while also ensuring that the right to culture as provided under the Constitution is respected. It also provides an opportunity for review and to address gaps that were not envisaged by the drafters of the anti-FGM/C law.

\subsection{Research: Validity of methods for data collection on FGM/C}

The two main approaches used in collecting data on FGM/C include self-reporting and physical examination of the genitalia. The debate on which of these approaches is the most suitable method has been longstanding, especially among programme implementers (Matanda 2020). The genesis of the debate is that in communities that practise $\mathrm{FGM} / \mathrm{C}$, it is a highly sensitive topic and open discussions are often considered a taboo. In situations where $F G M / C$ is illegal, people may be 
afraid to speak about FGM/C for fear of prosecution. Physical examination of the female genitalia as a method of collecting data on FGM/C is rarely used because of the ethical challenges and the associated costs.

Findings from the Evidence to End FGM/C Research Programme highlight the importance of being conscious of the specific strengths and limitations of each approach (Matanda 2020). Self-reports are less intrusive, ideal for reaching a larger population through surveys, and effective in documenting forms of FGM/C that cannot be identified through physical exams. Nevertheless, the approach is constrained by the challenges of reconciling the interviewer's versus interviewee's definition of what constitutes $\mathrm{FGM} / \mathrm{C}$, and the reduced reliability of self-reported data in settings where FGM/C is illegal. Physical examinations, if integrated into routine health services at health facilities, provide opportunities for prevention and monitoring of $\mathrm{FGM} / \mathrm{C}$ incidences, and identification and treatment of FGM/C-related complications. Nonetheless, in Kenya, where healthcare providers are required to report FGM/C cases to authorities, care should be taken to ensure that FGM/C survivors do not avoid routine visits to health facilities because of fear of prosecution. Provision of protocol on how physical examinations are to be conducted, enhancement of the capacity of the health care system to document and report FGM/C cases, and training of healthcare providers will be critical in facilitating collection of FGM/C data through clinical exams.

\section{Summary}

Over the last five years, the Evidence to End FGM/C Research Programme conducted research in Kenya with the aim of generating evidence to inform policy and programmes. Various studies were undertaken and covered contemporary aspects related to FGM/C including the motivations that people have for obeying the anti-FGM/C law; changes in the practice and social norms associated with $\mathrm{FGM} / \mathrm{C}$; the extent of medicalisation of $\mathrm{FGM} / \mathrm{C}$ and the health systems' response to prevention and management of FGM/C; and the use of data in mapping out FGM/C hotspot areas. The programme generated rich data that informed the following key findings and recommendations:

- While criminalisation of FGM/C has instilled fear of the law, it has not had a strong deterrent effect. Instead, criminalisation has driven the practice underground, and led to performing $\mathrm{FGM} / \mathrm{C}$ on younger girls supported through medicalisation. We recommend that the legal framework focus more on regulation than on criminalisation. Additionally, the law needs to be supported by community-led discussion of the practice and initiatives in the health and education sectors.

- There are substantial changes in how FGM/C is practised such as cutting at younger ages, less severe forms of cutting, and cutting in secret. There are also changes in social

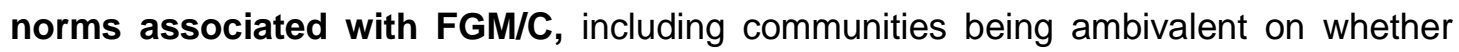
$\mathrm{FGM} / \mathrm{C}$ is a prerequisite for marriage, and whether cut girls are considered respectful or not depending on their ethnic background. Therefore, programme implementers and policymakers should pay attention to these changes and more research conducted to inform the design of interventions in order to accelerate and support abandonment. Research is specifically needed into the potential for social media in ending FGM/C given its rapid growth in Kenya.

- There is a trend towards health professionals performing FGM/C (medicalisation) in Kenya. Additionally, the health professionals lack the capacity and skills to prevent and 
manage FGM/C-related complications. The demand for medicalised FGM/C needs to be addressed by engaging community and religious leaders. The supply side should be addressed through professional dialogues and training to stop health professionals from performing $\mathrm{FGM} / \mathrm{C}$. Health system strengthening in response to $\mathrm{FGM} / \mathrm{C}$ should involve fidelity of legal/policy implementation, training of providers, funding and resource allocation, data capture/documentation, and integration of FGM/C into health programmes.

In-depth analysis of nationally representative survey data (KDHS) for girls aged 0-14 years provided a rich and detailed picture of when, where, and how FGM/C has been carried out, and trends in changes in the practice. However, challenges exist in the generation, access and usage of quality FGM/C data. There is need for innovative use of national surveys to assist in pinpointing where resources should be directed, and effective local interventions implemented. Evaluation of FGM/C interventions and monitoring of change is critical and should be prioritised by policymakers and programme implementers. This will enable sharing of knowledge on what works; lessons learned; and how to adapt, replicate, and scale up successful interventions.

\section{References}

28TooMany. 2018. "Kenya: The Law and FGM." London: 28 Too Many. Accessed 25 April 2020. Available at: https://www.28toomany.org/static/media/uploads/Law\%20Reports/kenya_law_report_v1_( may_2018).pdf

Abuya, K. 2020. "Embracing partnership for social transformation and the realization of human rights: A pathway to ending female genital mutilation, marginalization of women in political leadership, and other harmful practices amongst the Abagusii of Kenya," Interdisciplinary Journal of Partnership Studie. 7(1): 7.

Berg, R.C. and E. Denison. 2012. "Effectiveness of interventions designed to prevent female genital mutilation/cutting: A systematic review," Studies in Family Planning 43(2):135-146.

Cislaghi, B. and L. Heise. 2019. "Using social norms theory for health promotion in low-income countries," Health Promotion International 34(3): 616-623.

Cislaghi, B., D. Gillespie, and G. Mackie. 2016. "Analysis: How values deliberations lead to community empowerment," in: B. Cislaghi, D. Gillespie, and G. Mackie (eds.). Values Deliberation and Collective Action: Community Empowerment in Rural Senegal. Cham: Springer International Publishing, pp. 143-186.

Commonwealth Education Hub. 2016. "Female Genital Mutilation: The Role of Education." London: The Commonwealth Secretariat. Accessed 25 April 2020. Available at:

https://www.thecommonwealth-educationhub.net/wp-content/uploads/2016/07/FGM-andEducation-Brief_v2.pdf

Gruenbaum, E. 2001. The Female Circumcision Controversy: An Anthropological Perspective. Philadelphia: University of Pennsylvania Press.

2005. "Socio-cultural dynamics of female genital cutting: Research findings, gaps, and directions," Culture, Health \& Sexuality 7(5): 429-441.

Kandala, N-B., D. Kinyoki, A. Sarki, D. Gathara, and B. Shell-Duncan. 2017. "Modelling and Mapping of Girls' Female Genital Mutilation/Cutting (FGM/C) in the Context of Economic, 
Social and Regional Disparities from Kenya Demographic and Health Surveys, 19982014." Technical Paper for Evidence to End FGM/C: Research to Help Girls and Women Thrive. New York: Population Council.

Kandala, N-B., P. Komba, C.C. Nnanatu, G. Atilola, L. Mavatikua, Z. Moore, et al. 2020a. Modelling and Mapping of Regional Disparities Associated with Female Genital Mutilation/Cutting Prevalence Among Girls Aged 0-14 Years in Senegal: Evidence from Senegal (SDHS) Surveys 2005-2017. Evidence to End FGM/C: Research to Help Girls and Women Thrive. New York: Population Council.

Kandala N-B., P. Komba, C.C. Nnanatu, G. Atilola, L. Mavatikua, Z. Moore, et al. 2020b. Modelling and Mapping of State Disparities Associated with Female Genital Mutilation/Cutting Prevalence Among Girls Aged 0-14 Years in Nigeria: Evidence from DHS and MICS 2003-2017. Evidence to End FGM/C: Research to Help Girls and Women Thrive. New York: Population Council.

Kimani, S. and C. Kabiru. 2018. Shifts in Female Genital Mutilation/Cutting in Kenya: Perspectives of Families and Health Care Providers. New York: Population CouncilAccessed 29 April 2020. Available at: https://knowledgecommons.popcouncil.org/departments_sbsr-rh/561

Kimani, S. and O. Obianwu. 2020. Female Genital Mutilation/Cutting (FGM/C): Review of Laws and Policy Documents in Kenya and Nigeria. New York: Population Council.

Kimani, S. and C. Okondo. 2020. A Diagnostic Assessment of the Health System's Response to Female Genital Mutilation/Cutting Management and Prevention in Kenya. New York: Population Council. Accessed 24 April 2020. Available at: https://www.popcouncil.org/uploads/pdfs/2020RH_FGMC-DiagnosticAssessmentKenya.pdf

Kimani, S. and B. Shell-Duncan. 2018. "Medicalized female genital mutilation/cutting: Contentious practices and persistent debates," Current Sexual Health Reports 10(1): 2534.

Kimani, S., C. Kabiru, J. Muteshi, and J. Guyo. 2020. "Exploring barriers to seeking health care among Kenyan Somali women with female genital mutilation: A qualitative study," BMC International Health and Human Rights 6: 1-12.

Kimani, S., T. Esho, V. Kimani, S. Muniu, J. Kamau, C. Kigondu, et al. 2018. "Female genital mutilation/cutting: Innovative training approach for nurse-midwives in high prevalent settings" Obstetrics and Gynecology International March: https://doi.org/10.1155/2018/5043512

Mackie, G., J. LeJeune. 2009. "Social Dynamics of Abandonment of Harmful Practices: A New Look at the Theory." Innocenti Working Papers. Florence: UNICEF Innocenti Research Centre.

Matanda, D. 2020. Measurement of Female Genital Mutilation/Cutting Status: Perspectives from Healthcare Providers, Policymakers, Programme Implementers and Researchers. Evidence to End FGM/C: Research to Help Girls and Women Thrive. New York: Population Council

Matanda, D., C. Okondo, C. Kabiru, and B. Shell-Duncan. 2018. Tracing Change in Female Genital Mutilation/Cutting: Shifting Norms and Practices among Communities in Narok and Kisii Counties, Kenya. Evidence to End FGM/C: Research to Help Girls and Women Thrive. New York: Population Council. 
Meroka-Mutua, A., D. Mwanga, and O.C. Olungah. 2020. Assessing When and How Law Is Effective in Reducing the Practise of FGM/C in Kenya. Evidence to End FGM/C: Research to Help Girls and Women Thrive. New York: Population Council.

Ministry of Education (MOE). 2018. National School Heath Policy. Nairobi: MOE.

Ministry of Health (MOH). 2007. Management of Complications: Pregnancy, Childbirth and the Postpartum Period in the Presence of FGM/C. Nairobi: MOH.

Ministry of Public Health. 2009. National Guidelines on Management of Sexual Violence in Kenya. Nairobi: Ministry of Public Health.

Moses, B.G. 2008. Thinking the Gusii Way: Insider Perspectives on Female Genital Mutilation(FGM)/Cutting and Strategies for Change. Saarbrucken, Germany: VDM Verlag.

National Bureau of Statistics-Kenya (KNBS) and ICF International. 2014. Kenya Demographic and Health Survey 2014. Calverton, MD: KNBS \& ICF International.

OHCHR, UNAIDS, UNDP, UNECA, UNESCO, UNFPA, et al. 2008. "Eliminating Female Genital Mutilation: An Interagency Statement. 2008. Geneva: WHO.

Population Council - Evidence to End FGM/C: Research to Help Girls and Women Thrive Research Programme. 2020. Guidance Note. Improving the Rigor, Relevance and Utility of Descriptions of FGM/C Interventions and Their Evaluation. New York: Population Council.

The Republic of Kenya (ROK). 2011. "Prohibition of Female Genital Mutilation Act No 32 of 2011." Nairobi: National Council of Law Reporting (NCLR Kenya) with the Authority of the Attorney-General. Accessed 25 April 2020. Available at:

http://kenyalaw.org/kl/fileadmin/pdfdownloads/Acts/ProhibitionofFemaleGenitalMutilationAct _No32of2011.pdf

The Republic of Kenya (ROK). 2010. "The Constitution of Kenya, 2010." Kenya Law Reports (February): 31.

The Republic of Kenya (ROK). 2019. "National Policy for the Eradication of Female Genital Mutilation." Nairobi: Ministry of Public Service, Youth and Gender.

Shell-Duncan, B., D. Gathara, and Z. Moore. 2017. Female Genital Mutilation/Cutting in Kenya: Is Change Taking Place? Descriptive Statistics from Four Waves of Demographic and Health Surveys. New York: Population Council.

Shell-Duncan, B., R. Naik, and C. Feldman-Jacobs. 2016. A State-of-Art-Synthesis of Female Genital Mutilation/Cutting: What Do We Know Now? New York: Population Council.

United Nations (UN). 1979. Convention on the Elimination of all Forms of Discrimination Against Women. New York: United Nations Human Rights Office of The High Commissioner. Accessed 25 April 2020. Available at: https://www.ohchr.org/en/professionalinterest/pages/cedaw.aspx 2015. The Sustainable Development Goals (SDGs). New York: United Nations. Accessed 28 April 2020. Available at: https://sustainabledevelopment.un.org/sdg5

United Nations Population Fund (UNFPA) and United Nations Children's Fund (UNICEF). 2018. 2017 Annual Report: UNFPA-UNICEF Global Programme to Accelerate Action to End Child Marriage. New York: UNFPA-UNICEF. 
World Health Organization (WHO). 2018. Care of Women and Girls Living with Female Genital Mutilation: A Clinical Handbook. Geneva: World Health Organization. Accessed 21 April 2020. Available from: http://www.who.int/reproductivehealth/publications/health-care-girlswomen-living-with-FGM/en/

2020. The Economic Cost of Female Genital Mutilation. Geneva: WHO. Accessed 23 April 2020. Available at: https://www.who.int/news-room/detail/06-02-2020-economic-cost-offemale-genital-mutilation

Suggested Citation: Matanda, Dennis, Agnes Meroka-Mutua, and Samuel Kimani. 2020. "Evidence to End Female Genital Mutilation in Kenya: What We Have Learned from a FiveYear Research Programme and its Relevance for Policy and Programmes." Evidence to End FGM/C: Research to Help Girls and Women Thrive. New York: Population Council.

Acknowledgements: Many thanks to Bernadette Loloju, Jim Drummond, Baker Maggwa, Hazel Barrett, Jacinta Muteshi, and Michelle Hindin for reviewing and providing useful comments on earlier drafts of this synthesis report. 